\title{
Investigación en Progreso: Estudio y Evaluación de Tecnologías de la Información y la Comunicación para el Desarrollo de Ciudades Inteligentes
}

\author{
Hector Luis Vivas, Paola V. Britos, Nicolas García-Martinez, Mauro Cambarieri \\ Laboratorio de Informática Aplicada \\ Universidad Nacional de Rio Negro \\ Viedma, Argentina \\ hlvivas@unrn.edu.ar,pbritos@unrn.edu.ar
}

\begin{abstract}
Resumen - Los avances tecnológicos, la globalización, la demanda social para poder disponer de diversos servicios, la evolución en las comunicaciones, y el impulso político, han provocado que nos encontremos frente a una sociedad en la cual se generan cambios significativos en la forma de trabajar, de aprender, de pensar, de comunicarse y de vivir afectando a los ciudadanos a todos los niveles. Por esto, la forma en la que concebimos a las ciudades tradicionales se encuentra cambiando a lo que se conoce como ciudades inteligentes; las ciudades ahora comienzan a ser vistas como medios innovadores que ayudan al desarrollo y al progreso de las regiones e inclusive del país del que forman parte, logrando así una integración de las diversas áreas urbanas hacia la Sociedad de la Información. En nuestra región (Provincia de Rio Negro - Argentina) se ha podido identificar la necesidad de contar con desarrollos innovadores de tecnología para transformar dichas áreas urbanas en ciudades inteligentes. Esta evolución se verá apalancada por el aprovechamiento de las siguientes tecnologías: Televisión Digital Terrestre (TDT), Soluciones móviles, Internet de las Cosas (M2M), Plataformas Educativas Virtuales (PEV). EI desafío de este proyecto es construir capacidades y herramientas de software para ciudades inteligentes, maximizando los beneficios en la aplicación correcta y eficiente de las citadas tecnologías. Como, así también, la aplicación de marcos referenciales de planificación y calidad durante el proceso de desarrollo y apropiación de dichos recursos.
\end{abstract}

Palabras Clave - Ciudades Inteligentes, B2B, Educación, TV digital, telefonía móvil

\section{ESTADO DE LA CUESTIÓN}

\section{A. Ciudades inteligentes}

Según la Comisión de Ciudades Digitales de AUTELSI Asociación Española de Usuarios de las Telecomunicaciones y de la Sociedad de la Informacion - (2006) "Una ciudad digital es el espacio virtual de interacción entre todos los actores que participan en la vida de una ciudad (ciudadanos, empresas, administraciones, visitantes, etc.) utilizando como soporte los medios electrónicos y las tecnologías de la información y comunicación (TICs), ofreciendo a dichos actores acceso a un medio de relación y comunicación innovador, a través del canal que elijan, en cualquier momento y lugar. El objetivo principal es la mejora de la relación y los servicios entre los actores que interactúan en la ciudad, tanto en los servicios existentes como en los futuros, potenciando un desarrollo sostenible económico y social de la ciudad..."

En estos espacios virtuales interactúan los ciudadanos (habitantes, empresas, entidades, organismos, etc.) y la administración pública utilizan las tecnologías de la información y la comunicación con el fin de mejorar sus relaciones de la vida diaria, a través del gobierno electrónico (e-government), teletrabajo (e-work), comercio electrónico (ecommerce), teleentretenimiento (e-entertaiment), de la telesalud (e-health), teleeducación (e-education), entre otros, impulsando el desarrollo de la Sociedad la Información. Una Ciudad Digital tiene las mismas características de una ciudad real constituida, con la diferencia de que la ciudad digital tiene "virtualidad" y persigue contribuir, en forma más ágil y eficiente, a una mayor participación de la ciudadanía y al mejoramiento de la calidad de vida de sus habitantes, coadyuvando de esta manera al progreso económico y social de la ciudad real. Los servicios que se ofrecen en las Ciudades Inteligentes se pueden clasificar en tres grandes grupos:

- Servicios de Comercio Electrónico: Comercio electrónico B2B y B2C, eLearning, GeoMarketing, Logística, Contenidos digitales (tv vía cable, tv on demand, banda ancha, etc.), Servicios en cadena provistos para PyMEs.

- Servicios al Ciudadano: eGoverment, Educación, Medio Ambiente, Salud y Sanidad, Comunidades, Agencia de Trabajo, Entretenimiento, Arte, Cultura.

- Servicios de Acceso Libre, que ofrecen información y comunidades acerca de: Salud y bienestar, Religión, Drogas, Aficiones, Educación y formación profesional, Tercera edad.

Según la AUTELSI, en Iberoamérica, son cada vez más las ciudades que se suman a la digitalización con el objetivo de minimizar las brechas económica, social y cultural que todavía separan a la región de los países más avanzados. Se pueden distinguir dos tipos de actividades. Por un lado, las iniciativas que organizaciones como AHCIET (Asociación Iberoamericana de Centros de Investigación y Empresas de Telecomunicaciones) despliegan a favor de esta causa: foros, eventos, estudios o proyectos de digitalización. La AHICIET destaca prácticas sobre esta temática en las siguientes ciudades de Latinoamérica:

- Guadalajara (México), premiada por su transparencia, impulso de los negocios, desarrollar una Intranet que conecta a toda la Administración Municipal y reducir el número de quejas por falta de transparencia al publicar toda la información relacionada con la localidad en su web. También posee un portal específico para negocios: Bussines Guadalajara Metropolitan, así como un programa de desarrollo de emprendedores (capacitación y financiamiento para pequeños negocios). El municipio mexicano de Monterrey, donde la población tiene acceso a Internet en las 
escuelas, dispone de parques y plazas públicas con acceso WiFi gratuito y de 5 Centros de Atención Ciudadana. Además, se han instalado cámaras de vigilancia urbana colgadas de una red inalámbrica WiMax propiedad del municipio y un botón de pánico conectado de manera inalámbrica al sistema de respuesta inmediata, entre otras acciones.

- Recoleta (Chile). Es un exponente de integración de discapacitados y de cooperación público-privada, desarrollando tecnologías ad hoc para no videntes. Han firmado acuerdos entre el Ayuntamiento y otros entes públicos y privados para la capacitación de microempresarios. Dispone de una Zona Pyme - espacio para los empresarios- y una Fundación web dotada con recursos económicos para ayudar a los pequeños empresarios.

- Localidades de Perú, Chile, Colombia y Argentina también se han destacado por su trabajo a favor de la Sociedad de la Información, lo que deja entrever la importancia que se concede a este tema.

\section{B. Televisión Digital}

La Televisión Digital Terrestre o TDT es una plataforma de transmisión de señales de televisión moderna y más eficiente que la actual emisión analógica. En la Argentina nació por iniciativa del Gobierno Nacional y se encuentra en pleno proceso de expansión. La misma implica una forma distinta de transmitir la señal televisiva, diferente a la televisión analógica actual. El 31 de agosto de 2009 se creó el Sistema Argentino de Televisión Digital Terrestre (SATVD-T), el cual consiste en un conjunto de patrones tecnológicos a ser adoptados para la transmisión y recepción de señales digitales terrestres, radiodifusión de imágenes y sonido [1]. Su evolución, permite decir al Gobierno Nacional que "en el 2019 se producirá el Apagón Analógico", frase que nos permite inferir que los próximos 7 años estarán marcados por una rápida evolución en los formatos, los contenidos y las formas en que interactuamos con la actual televisión. [1].

Podemos observar que según la forma de recepción de la señal en el hogar, se puede hablar de Televisión Digital por Satélite, Televisión Digital por Cable o Televisión Digital Terrestre. Las dos primeras tecnologías están presentes en Argentina, hasta hace poco tiempo únicamente brindadas por empresas privadas. La creación del Sistema Argentino de Televisión Digital Terrestre cambia el escenario nacional, ofreciendo televisión abierta, libre y gratuita con mejores prestaciones que podrá ser vista en todo el territorio nacional.

Se puede mencionar que la Republica Argentina optó por el Sistema Brasileño de Televisión Digital Terrestre (SBTVDTB), basado en el sistema Japonés denominado ISDB-T. El mismo permite la recepción terrestre, portátil y móvil de la señal transmitida. La norma ISDB-T fue tomada prácticamente por todos los países del cono sur, con lo cual permitirá la integración, el desarrollo cultural y tecnológico en el conjunto de los países de Latinoamérica. La norma ISDB-T permite la posibilidad de tener interactividad, transformando al receptor en un dispositivo bidireccional que permitirá al televidente tomar un rol activo, con capacidad para relacionarse con los contenidos televisivos. [1], [2]. Otra de las fortalezas de la norma japonesa es la posibilidad de transmitir TV gratuita para dispositivos móviles. El modelo que llega al celular es el denominado "One-seg", implementado únicamente por esta norma, que utiliza parte de la trama de la señal digital asignándole a un segmento la difusión de la señal televisiva. Un receptor de "One-seg" se instala fácilmente en teléfonos celulares, sintonizadores USB de notebooks, tablet, etc. con lo cual mirar televisión es gratuito, también desde dispositivos móviles. [3], [4].

En el sistema de Televisión Digital Terrestre, el middleware es un componente fundamental para la recepción de aplicaciones interactivas en el lado del receptor (Set Top Box) o televisores digitales con el sintonizador incluido. El Middleware es una capa de software intermedia entre el hardware/sistema operativo y las aplicaciones, que ofrece una serie de facilidades para el desarrollo de contenidos interactivos para TDT. Existen diferentes middleware para la televisión digital terrestre: middleware DASE (Americano), middleware MHP (Europeo), middleware ARIB (Japonés) y el middleware Ginga (Brasileño). Ginga ${ }^{\circledR}$ es el nombre del Middleware Abierto del Sistema Brasileño de TV Digital (SBTVD). Ginga es software libre porque desde su concepción, tuvo en consideración la necesidad de la inclusión social/digital y la obligación de compartir el conocimiento de forma libre. El middleware Ginga está compuesto por dos entornos de trabajo, definidas como Ginga-NCL (Nested Context Language) y Ginga-J (Java); ambos definidos en dos lenguajes; el primero de tipo declarativo y el segundo de forma procedimental. El entorno declarativo de Ginga, llamado Ginga-NCL tiene una implementación de referencia en código abierto, desarrollada por el Laboratorio TeleMídia de la Pontificia Universidad Católica de Rio de Janeiro - PUC-Rio. Adoptando la licencia GPLv2, el laboratorio TeleMídia garantiza el acceso permanente a toda la evolución del código publicado en la Comunidad Ginga, sean cuales fueren sus aplicaciones y autores de aquí en adelante. A la fecha existen numerosos proyectos de investigación relacionados al Ginga. Explícitamente al Ginga-NCL, ya que es un estándar aceptado por la ABNT (Asociación Brasileña de Normas Técnicas) y a nivel internacional.

\section{Telefonía Móvil}

Como hace algo más de una década sucedió con Internet, la movilidad es cada día una parte más importante de nuestras vidas $\mathrm{y}$ crece a un ritmo sin precedentes. Las primeras soluciones móviles vienen con la aparición de las notebook. Por sus limitaciones físicas, como tamaños, pocas horas de duración de batería, los llamados teléfonos inteligentes "smartphones", rápidamente penetraron en el mercado móvil, remplazando tempranamente a las notebook y netbook, en el segmento de soluciones móviles. [5]. Cuando hablamos de soluciones móviles, nos referimos a software que corren en smartphone o Tablet, como pueden ser juegos, comercio electrónico, redes sociales, servicios financieros, publicidad y Cloud Computing (Procesamiento en las nubes). Los primeros smartphone con cámara, wi-fi, pantallas táctiles, dieron la posibilidad de crear aplicaciones muy interesante para todo tipo mercado. El iPhone, creado por Apple, fue sin lugar a duda un punto de partida para estos dispositivos. Actualmente tenemos una amplia variedad de hardware móvil, como tablet, smartphone y phablet (una combinación entre Tabletas y teléfonos inteligentes). La diferencia de cada uno de estos dispositivos radica en el tamaño de la Pantalla. El incremento de la potencia de cálculo y bajo consumo de los nuevos APU y ARM, debido a los nuevos proceso de fabricación (40nm), posibilitan que los dispositivos cuenten con 4 Cores y GPU con posibilidades de juegos 3D, vídeo en 1080p, 
procesamiento de imagines en tiempo real y la posibilidad de crear cualquier tipo aplicación. Este tipo de hardware se presentó en Mobile World Congress (MWC), donde predominaron los teléfonos Android. [6]

En la actualidad, el hardware existente no es una limitación para crear aplicaciones móviles. Además del poder de cálculo, los dispositivos móviles cuentan una gran variedad de Hardware, como GPS para la geolocalización, sensores como giroscopio que detecta orientación, acelerómetros que permiten detectar los movimientos del dispositivo, sensor de proximidad y luz ambiental entre los más destacado.

Los smartphone de bajo costo serán uno de los desafíos más importante para el desarrollo y avance de la industria en las regiones emergentes [7]. Otro punto destacado como desafío es el desarrollo de estándares, para frenar la fragmentación de frameworks y plataformas de desarrollo, actualmente los más destacas son Java Me, Windows Mobile (basado en Win32), Cocoa Touch (iPhone) y Web basado en los estándares de Html. HTML5 podría ser el estándar a adoptar, plataformas como BlackBerry 10 y los smartphone de Mozilla trabajan en este rumbo. Actualmente iPhone y Android soportan el estándar HTML5. Las nuevas apuestas móviles para los próximos años, tienen como más destacado el negocio mHealth (Salud móvil) con una estimación para el 2017 de U\$ 23.000.000.000 [6]. Los nuevos dispositivos contarán con sensores de signos vitales, lo que permitirá monitoreo remoto de pacientes, además de cámaras de alta densidad para el proceso de imágenes en tiempo real y diagnóstico. Otra área derivada del mHealth, es Mobile Baby, con la finalidad de atacar la mortalidad de la madre y del recién nacido. Otro mercado es Dinero Móvil, a través de un componente de Hardware para móviles llamado NFC (Near Field Communication o Comunicación de campo cercano), una tecnología de comunicación inalámbrica, de corto alcance y alta frecuencia que permite el intercambio de datos entre dispositivos a menos de $10 \mathrm{~cm}$, cuya aplicación se utiliza para el intercambio de bienes y servicios. Se estima que para el 2014 los pagos a través de NFC alcanzarán los U\$ 500.000.000 [6].

El futuro de la movilidad está basado en cloud (Datos en las Nubes), la industria del móvil utilizará la Nube para consolidar su negocio corporativo. El modelo Cloud Computing es facilitador, eficiente y reduce costos totales propietarios. Los desafíos para las Nubes serán consolidar la conectividad y seguridad

\section{Machine to Machine o la Internet de las Cosas}

Machine to Machine (M2M) es la tecnología que apoya la comunicación inalámbrica entre dispositivos, También se utiliza para dar soporte entre un operado y un máquina. M2M inalámbrica comprende todas las tecnologías de redes inalámbricas, aunque el término es generalmente se utiliza en referencia a los sistemas conectados a las redes celulares. Este informe está dirigido a describir y analizar sistemas inalámbricos M2M basadas en uso común de la red inalámbrica de área amplia tecnologías tales como GSM / GPRS / EDGE, WCDMA / HSPA, CDMA y LTE. La tecnología M2M hasta llegar a la telemetría que es una tecnología que permite el control remoto medición y reporte de información de interés para el operador del sistema. En el pasado, los sistemas de telemetría eran del dominio exclusivo de grandes organizaciones. Las grandes petroleras y las compañías de gas y electricidad, fueron algunas de las primeras organizaciones en utilizar ampliamente telemetría. También las agencias espaciales utilizan la telemetría para controlar satélites y naves espaciales tripuladas.

Hoy M2M se utiliza para indicar el movimiento actual en el espacio de telemetría para llevar el concepto de adquisición de datos y control remoto a un público más amplio. El avance de la tecnología, con la mejora de las capacidades y la cobertura de las redes inalámbricas así como aceleración de la adopción de sistemas tecnológicos en toda la sociedad, está haciendo inalámbrica, a disposición de todas las organizaciones en el mundo industrializado. En el espacio de unas pocas décadas, la tecnología ha encontrado un gran número de aplicaciones en todas las industrias. M2M se utiliza en telemetría, recolección de datos, control remoto, la robótica, el control remoto, seguimiento de estado, por carretera, control de tráfico, fuera del área de diagnóstico y mantenimiento, sistemas de seguridad, servicios logísticos, gestión de flotas y la telemedicina. Con frecuencia las tecnologías alámbricas e inalámbricas de comunicación competir para las mismas aplicaciones. Sin embargo, inalámbrica es la única opción cuando la movilidad es necesaria - por ejemplo, en aplicaciones de automotrices. Otra de las ventajas de la tecnología inalámbrica a través de redes es la adaptabilidad a diferentes ambientes, gracias a la disponibilidad de las redes celulares en todas las zonas pobladas. [8].

\section{E. Plataformas Educativas}

Con la integración de la tecnología a nuestra forma de vida, es necesario plantear nuevas formas de interacción entre personal docentes y alumnos. Pero es tarea de importancia prioritaria que los profesores plasmen el contenido de forma eficaz, gestionando actividades de aprendizaje que contemplen los métodos clásicos con la ventaja de las aplicaciones de la Informática.

Lo que en gran medida nos lleva a pensar en la seguridad y garantías de aprendizaje de esta nueva estrategia, cabe resaltar que su instauración en los sistemas educativos es creciente debido a las demandas del mundo actual. Actualmente se utilizan diversas plataformas educativas dirigidas a procesos y ejecuciones en red de los métodos pedagógicos, entre los ejemplos que podemos encontrar están Web Course Tool (WebCT), Learningspace y Blackboard.

Es importante señalar que se debe ofrecer una herramienta que permita adaptación a las singularidades de cada metodología de estudio de las diversas áreas del conocimiento, ya que ésta es una de las piezas clave para la aceptación y rápida integración a los sistemas educativos [9].

Las plataformas educativas son en los entornos virtuales de aprendizaje y enseñanza (EVA) que forman un espacio de interacción entre el profesor y alumno, en el que el estudiante se puede comunicar de 2 maneras. La asincrónica (en tiempo y espacio distinto) ejemplo blogs, wikis, e mail y la sincrónica (diferentes espacios pero mismo tiempo) ejemplo chat, webam, videoconferencia.

Actualmente la mayoría de las universidades de todo el mundo cuentan con una plataforma virtual educativa que facilita la consulta de materiales educativos, pruebas en línea, publicaciones, avisos, envíos de tareas, comunicación ente profesores y alumnos.

Una de las plataformas mas confiables utilizando software libre es Moodle (Modular Object-Oriented Dynamic Learning Environment -Entorno de Aprendizaje Dinámico Orientado a 
Objetos y Modular) donde se pueden realizar todas las actividades pedagógicas relacionadas con la transmisión y distribución de contenido y materiales que se necesitan para llevar a cabo las actividades de una o varias materias. Cuenta con la posibilidad de agregar espacios de chateo, debate o de retroalimentación y estadísticas para grupos y la comunidad educativa en general. Esta plataforma es de uso libre, y es la más utilizada en este rubro, con un total de 809,906 usuarios al mes de Octubre de 2009. Varias universidades de gran prestigio en el mundo utilizan esta herramienta, por ejemplo el Instituto Politécnico Nacional cuenta con una plataforma llamada "Polivirtual", la cual utiliza Moodle, cuya principal característica es la transmisión en vivo de clases para propiciar el aprendizaje en línea.

\section{OBJetivos GeNERALES Y ESPECÍfICOS E HiPÓTESIS DE TRABAJO}

Es objetivo general de este proyecto:

- Desarrollar servicios electrónicos innovadores para ciudades inteligentes.

Sus objetivos específicos son:

- Identificar teorías, metodologías y modelos conceptuales para el desarrollo de ciudades inteligentes.

- Identificar tecnologías y soluciones innovadoras emergentes.

- Optimizar soluciones electrónicas para aplicar en la evolución de las ciudades inteligentes.

- Desarrollar y validar prototipos de software, pasibles de ser implementados y transferidos al medio.

- Comunicar los resultados obtenidos.

\section{RELEVANCIA DEL PROBLEMA}

La relación entre ciudad y tecnología ha existido desde comienzos de la vida urbana y social. Los avances tecnológicos, la globalización, la demanda social para poder disponer de diversos servicios, la evolución en las comunicaciones, y el impulso político, han provocado que nos encontremos frente a una sociedad en la cual se generan cambios significativos en la forma de trabajar, de aprender, de pensar, de comunicarse y de vivir afectando a los ciudadanos a todos los niveles. Por esto, la forma a en la que concebimos a las ciudades tradicionales se encuentra cambiando también a lo que se conoce como ciudades inteligentes; las ciudades ahora comienzan a ser vistas como medios innovadores que ayudan al desarrollo y al progreso de las regiones e inclusive del país del que forman parte, logrando así una integración del mismo hacia la Sociedad de la Información; sociedad en la que se comienza a competir por un lugar en una red global que conduce, según Castells (1996), a un espacio de flujos dentro del cual interactúan ciudades y regiones que buscan el progreso.

Según la Comisión de Ciudades Digitales de AUTELSI Asociación Española de Usuarios de las Telecomunicaciones y de la Sociedad de la Informacion - (2006) "Una ciudad digital es el espacio virtual de interacción entre todos los actores que participan en la vida de una ciudad (ciudadanos, empresas, administraciones, visitantes, etc.) utilizando como soporte los medios electrónicos y las tecnologías de la información y comunicación (TICs), ofreciendo a dichos actores acceso a un medio de relación y comunicación innovador, a través del canal que elijan, en cualquier momento y lugar. El objetivo principal es la mejora de la relación y los servicios entre los actores que interactúan en la ciudad, tanto en los servicios existentes como en los futuros, potenciando un desarrollo sostenible económico y social de la ciudad...". En estos espacios virtuales interactúan los ciudadanos (habitantes, empresas, entidades, organismos, etc.) y la administración pública utilizan las tecnologías de la información y la comunicación con el fin de mejorar sus relaciones de la vida diaria, a través del gobierno electrónico (e-government), teletrabajo (e-work), comercio electrónico (e-commerce), tele-entretenimiento (eentertainment), de la tele-salud (e-health), teleeducación (eeducation), entre otros, impulsando el desarrollo de la Sociedad la Información. Según la AUTELSI, en Iberoamérica, son cada vez más las ciudades que se suman a la digitalización con el objetivo de minimizar las brechas económica, social y cultural que todavía separan a la región de los países más avanzados. Las iniciativas que organizaciones como AHCIET (Asociación Iberoamericana de Centros de Investigación y Empresas de Telecomunicaciones) despliegan a favor de esta causa: foros, eventos, estudios o proyectos de digitalización. La AHICIET destaca prácticas sobre esta temática en ciudades de Latinoamérica, como Guadalajara (México), Recoleta (Chile) y algunas localidades de Perú, Chile, Colombia y Argentina.

Algunos de los casos locales a nuestra región, en los cuales se implementaron "soluciones" y herramientas orientadas en la dirección de una ciudad digital son:

- La ordenanza municipal No 1957-CM-09 del Concejo Municipal de San Carlos de Bariloche que Implementa el Sistema de Voto Electrónico (01/10/2009).

- La ley 4082 de la Provincial de Río Negro que incorpora el Voto Electrónico en la provincia (Exped. 472/07 Sancionado 08/11/2007).

- La ordenanza municipal $\mathrm{N}^{\mathrm{o}}$ 380-CM-94 del Concejo Municipal de San Carlos de Bariloche que establece la primera etapa de implementación del Boleto Electrónico (1994).

- La ordenanza municipal $\mathrm{N}^{\mathrm{o}}$ 608-CM-96 del Concejo Municipal de San Carlos de Bariloche que modifica la implementación del Estacionamiento Medido con Parquímetros Móviles y Cospel Electrónico (1996).

- La ordenanza municipal $\mathrm{N}^{\circ}$ 2284-CM-12 del Concejo Municipal de San Carlos de Bariloche que establece el sistema de Estacionamiento Medido en el Microcentro, de tipo electrónico con registración OnLine (2012).

- La presentación del proyecto de la Intranet Pública Provincial (IPP) en la provincia de Río Negro, basada en una Única Red de Datos que permitiría que los diferentes organismos públicos, empresas públicas, y beneficiarios de servicios públicos distribuidos a grandes distancias, se conecten a través de una Intranet administrada en forma centralizada.

Así, en nuestra región, se ha podido identificar la necesidad de contar con desarrollos que la conviertan en una ciudad digital, en las siguientes áreas:

- La Televisión Digital Terrestre o TDT es una plataforma de transmisión de señales de televisión moderna y más eficiente que la actual emisión analógica. En la Argentina nació por iniciativa del Gobierno Nacional y se encuentra en pleno proceso de expansión. La misma implica una forma distinta de transmitir la señal televisiva, diferente a la televisión analógica actual. El 31 de agosto de 2009 se creó el Sistema Argentino de Televisión Digital Terrestre (SATVD-T), el cual consiste en un conjunto de patrones tecnológicos a ser 
adoptados para la transmisión y recepción de señales digitales terrestres, radiodifusión de imágenes y sonido [1]. Su evolución, permite decir al Gobierno Nacional que "en el 2019 se producirá el Apagón Analógico", frase que nos permite inferir que los próximos 7 años estarán marcados por una rápida evolución en los formatos, los contenidos y las formas en que interactuamos con la actual televisión. [1].

- A este avance se le suman las soluciones móviles, que ya nos referimos a software que corren en diversos tipos de celulares como pueden ser juegos, comercio electrónico, redes sociales, servicios financieros, publicidad y Cloud Computing (Procesamiento en las nubes).

- Siguiendo a los avances tecnológicos que favorecen la inclusión en una ciudad digital se encuentra "el internet de las cosas" o "Machine to Machine (M2M)" que es la tecnología que apoya la comunicación inalámbrica entre dispositivos, también se utiliza para dar soporte entre un operador y una máquina y comprende todas las tecnologías de redes inalámbricas, M2M se utiliza en telemetría, recolección de datos, control remoto, la robótica, el control remoto, seguimiento de estado, por carretera, control de tráfico, fuera del área de diagnóstico y mantenimiento, sistemas de seguridad, servicios logísticos, gestión de flotas y la telemedicina. Con frecuencia las tecnologías alámbricas e inalámbricas de comunicación compiten para las mismas aplicaciones. Sin embargo, la inalámbrica es la única opción cuando la movilidad es necesaria - por ejemplo, en aplicaciones de automotrices -.

- Finalmente, con la integración de la tecnología a nuestra forma de vida, es necesario plantear nuevas formas de interacción entre ciudadanos y gobiernos, asegurando que los actores reúnan las capacidades para adoptar esas nuevas formas de comunicación y participación.

Para el desarrollo de esas capacidades es necesario que se plasmen conocimientos de forma eficaz, gestionando actividades de aprendizaje que contemplen los métodos clásicos con las ventajas de las aplicaciones de las nuevas tecnologías de la información y las comunicaciones.

En este contexto, el uso de plataformas educativas virtuales ofrece herramientas que permiten la adaptación a singularidades de cada ciudad, en diversas situaciones o problemáticas, permitiendo la construcción de conocimientos y desarrollo de habilidades en forma colaborativa.

En este contexto, el proyecto pretende resolver los siguientes interrogantes:

- ¿Qué aplicaciones de software basadas en TDT, telefonía móvil, internet de las cosas y plataformas virtuales se necesitan en la región, de forma tal que permitan la integración de la población a una ciudad inteligente, favoreciendo la inclusión de los ciudadanos en la Sociedad de la Información?

- ¿Qué capacidades, tanto humanas como institucionales se requieren en la región para que una ciudad tradicional pueda evolucionar a una ciudad inteligente y los ciudadanos mejoren su calidad de vida a partir de la mejora en la prestación de los servicios por parte del estado y su articulación con las políticas públicas?.
[2] Stallings, W. 2000. Comunicaciones y Redes de Computadores. 6ta. Edición. Ed. Pearson Prentice Hall.

[3] Sklar, B. 2001. Digital Communications- Fundamentals and Applications. Ed. Prentice Hall.

[4] Tomasi, W. 2003. Sistema de Comunicaciones Electrónicas. 4ta. Edición. Ed. Prentice Hall.

[5] TSMC. 2012. Proceso de Fabricación ARM Cortex 28nm. http://www.tsmc.com/tsmcdotcom/PRListing NewsAction.do? action $=$ detail\&newsid $=6781$ \&language $=$ E. Ultimo acceso Julio 2012.

[6] InformationTecnology.2012 Mundo Móvil. Revista $N^{\circ} 174$

[7] Rodríguez Palchevich, D; González Terán, Y; Moreno, D. 2011. Estado del arte de la lectura en dispositivos móviles en los profesionales de información de la República Argentina. http://eprints.rclis.org/bitstream/10760/16494/1/lectura-movilargentina.pdf. Ultimo acceso Agosto 2012.

[8] yberg, T. 2010. The Global Wireless M2M Market. Third Edition, M2M Research Series 2010

[9] Rodríguez. Diéguez. Saénz Barrio. Tecnología Educativa y Nuevas tecnologías aplicadas a la educación. Alcoy, Marfil.

[10] Romero, A.; Fajardo, R. 2003. Diseño y desarrollo de una plataforma virtual de aprendizaje para la educación a Distancia.bwww.tise.cl/archivos/tise2003/papers/diseno_desarro llo_de_una_plataforma_virtual.pdf. Ultimo acceso Julio 2010 .

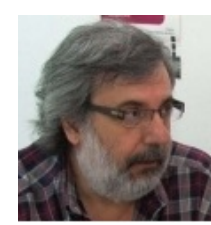

Luis Vivas. Es Licenciado en Análisis de Sistemas. Master en Informática. Master in Project Management. Profesor Asociado Regular en Ingeniería del Software. Coordinador de la Licenciatura en Sistemas. Director del Laboratorio de Informática Aplicada. Co-Director del proyecto de investigación "Estudio y evaluación de tecnologías de la información y la comunicación para el desarrollo de ciudades inteligentes" de la Universidad Nacional de Río Negro. $\mathrm{Su}$ interés en la investigación e centra en ciudades inteligentes.

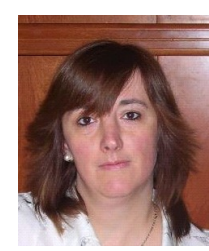

Paola Britos. Es Licenciada en Sistemas de Información por la Universidad Nacional de Luján, Magister en Ingeniería del Conocimiento por la Universidad Politécnica de Madrid y Doctora en Ciencias Informáticas por la Universidad Nacional de La Plata. Es Profesora Asociada Regular del Área de Ingeniería de Software en la Licenciatura en Sistemas de Información y Directora de los proyectos 40B133 y 40B065 de la Universidad Nacional de Río Negro. Sus intereses en investigación son modelos de proceso para proyectos de explotación de información.

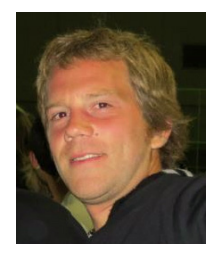

Nicolas Garcia-Martinez. Ingeniero en Sistemas de Información en la Universidad Tecnológica Nacional, Facultad Regional Rosario en 2009. Docente Interino con categoría de ayudante de Primera con dedicación completa en los espacios curriculares de "Introducción a las Bases de datos I", "Introducción a las Bases de datos II", "Bases de dates I" y "Bases de datos II" de la carrera de Licenciatura en Sistemas, en Sede Atlántica, Viedma.

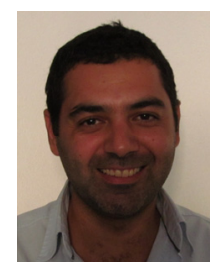

Mauro Cambariei. Ingeniero en Sistemas de Información en la Universidad Tecnológica Nacional (Regional La Plata). Actualmente trabaja en la Universidad Nacional de Río Negro en la carrera Licenciatura en Sistemas, se desempeño como JTP a cargo de las materias: Orientación a Objetos I, Seminario de Lenguajes Java, Base de datos II y Proyecto de Software. También forma parte del Laboratorio de Informática aplicada (UNRN) donde desempeña funciones de investigación y desarrollo en nuevas tecnologías. Su investigación dentro del laboratorio se centra en el desarrollo de software para televisión digital y desarrollo avanzado de aplicaciones empresariales a través de Internet.

\section{REFERENCES}

[1] Simoneta, J. 2002. Televisión Digital Avanzada. 1er. Edición. Ed. Intertel. 